\title{
Quantitative conditional quantum erasure in two-atom resonance fluorescence
}

\author{
Matthias Jakob ${ }^{1,3}$ and János Bergou ${ }^{1,2}$ \\ ${ }^{1}$ Department of Physics, Hunter College, City University of New York, 695 Park Avenue, New York, NY 10021, USA \\ ${ }^{2}$ Institute of Physics, Janus Pannonius University, H-7624 Pécs, Ifjúság útja 6, Hungary and \\ ${ }^{3}$ Department of Physics, Royal Institute of Technology (KTH), \\ SCFAB, Roslagstullsbacken 21, S-10691 Stockholm, Sweden
}

(Dated: January 2, 2019)

\begin{abstract}
We present a conditional quantum eraser which erases the a priori knowledge or the predictability of the path a photon takes in a Young-type double-slit experiment with two fluorescent four-level atoms. This erasure violates a recently derived erasure relation which must be satisfied for a conventional, unconditional quantum eraser that aims to find an optimal sorting of the system into subensembles with particularly large fringe visibilities. The conditional quantum eraser employs an interaction-free, partial which-way measurement which not only sorts the system into optimal subsystems with large visibility but also selects the appropriate subsystem with the maximum possible visibility. We explain how the erasure relation can be violated under these circumstances.

PACS numbers: 03.65.Ta, 03.65.Ud, 03.67.-a
\end{abstract}

\section{INTRODUCTION}

The quantum eraser [1] has been approved as a remarkable tool for studying fundamental topics in quantum mechanics. Among them we mention complementarity [2, the understanding of measurements in quantum mechanics, and, most importantly, the entanglement between subsystems which enables the very concept of quantum

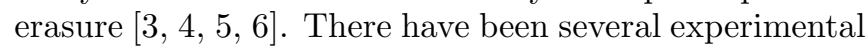
realizations of the quantum eraser demonstrating complementarity as one of the most basic principles of quantum mechanics 17, 8, 9, 10, 11, 12]. Further, quantitative measures of complementarity have been derived on the basis of an inequality for (partial) predictability $\mathcal{P}$ of the path the particle takes and (partial) visibility $\mathcal{V}$ of the interference fringes in a two-way interferometer [13, 14, 15] and experimentally verified [16]. Somewhat later, Englert 117 as well as Jaeger, Shimony, and Vaidman [18] derived an inequality which quantifies complementarity in a two-way interferometer supplemented by a which-way detector. They introduce the new quantity distinguishability $\mathcal{D}$ which quantifies the maximum possible information, obtainable by the which-way detectors, that Nature can grant us about the path the particle actually takes. The inequality has also been verified in several subsequent experiments [19, 20]. It has been recognized that the which-path knowledge $\mathcal{K}$ which is the practically available which-way information granted by the which-way detectors may be considerably less than the distinguishability $\mathcal{D}$ in realistic which-path measurements [21, 22, 23].

The inequalities have been extended in order to incorporate quantum erasure by introducing the quantum eraser visibility, $\mathcal{V}^{(\mathrm{QE})}$ 23], or the equivalent conditioned visibility, $\mathcal{V}_{c}$ [21]. The results can be summarized by the erasure relation [23]

$$
\mathcal{P}^{2}+\mathcal{C}^{2} \leq 1
$$

where $\mathcal{C}$ is the coherence which denotes the maximum possible quantum eraser visibility, $\mathcal{V}^{(\mathrm{QE})} \leq \mathcal{C}$, Nature can grant us about the visibility of the interference fringes. Taking into account that the smallest value of the actual which-path information, $\mathcal{K}$, is given by the a priori knowledge, $\mathcal{P}$, the following inequality inequality has been derived [23],

$$
\mathcal{K}^{2}+\left(\mathcal{V}^{(\mathrm{QE})}\right)^{2} \leq 1
$$

which states that the quantum eraser visibility $\mathcal{V}^{(\mathrm{QE})}$ can not exceed the maximum value $\left(1-\mathcal{P}^{2}\right)^{1 / 2}$, since $\mathcal{P} \leq \mathcal{K}$. Thus, a conventional quantum eraser can not erase the a priori predictability $\mathcal{P}$ which fundamentally limits the performance of quantum erasure.

In this paper we demonstrate erasure of the a priori predictability with a conditional quantum eraser. The retrieved visibility explicitly exceeds the maximum possible value derived for conventional quantum erasers [see Eq. (1.1)]. The conditional quantum eraser not only does sort subensembles as conventional quantum erasers do but, beyond it, also selects a proper subensemble. This can be achieved by employing interaction-free partial measurements, as has been suggested in the paper by Elitzur and Dolev [24] who consider nonlocal effects of partial measurements and quantum erasure. We extend their results to erasure of a priori knowledge and derive the underlying physical concept which enables predictability to be erased and which turns out to be concurrence or two-particle visibility. The concept of concurrence and especially two-particle visibility yields an interesting relation between entanglement and complementarity. Further, the quality of the recovered interference fringes will strongly depend on the amount of concurrence in the initial system. The partial interaction-free measurement composes a non-unitary transformation which, evidently, has a certain probability of failure. However, we can always and unambiguously tell whether the transformation (erasure) has succeeded.

We implement the conditional quantum eraser in an interference experiment with light scattered from two 


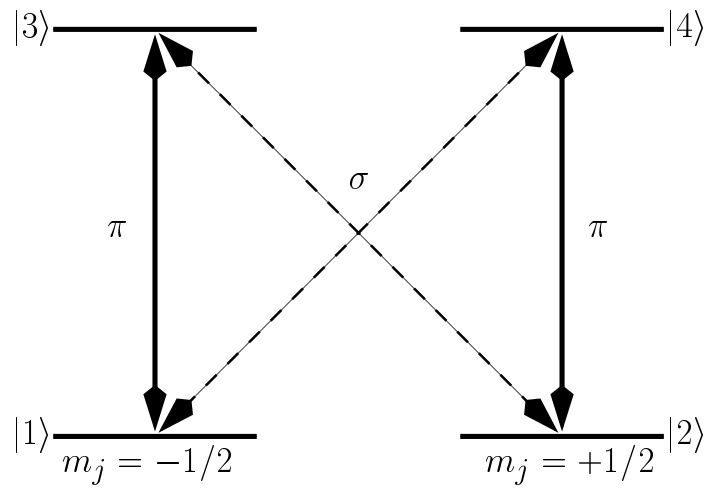

FIG. 1: Internal structure of the four-level atom with relevant polarization-sensitive transitions. Transitions between the states $|1\rangle \leftrightarrow|3\rangle$ and $|2\rangle \leftrightarrow|4\rangle$ which preserve the internal magnetic quantum number $m_{j}$ are connected with $\pi$ or $z$-polarized light, while transitions between $|1\rangle \leftrightarrow|4\rangle$ and $|2\rangle \leftrightarrow|3\rangle$ change the magnetic quantum number and lead to $\sigma$ - or $x$ - and $y$-polarized scattering events.

trapped four-level atoms. The internal structure of the four-level atom together with polarization-sensitive transitions is displayed in Fig. 1 (see also Ref. [25]). Recently, this remarkable Young-type double-slit experiment succeeded in the observation of polarization-dependent interference effects [26] and stimulated ongoing research [27]. We model the partial interaction-free measurement with the help of inefficient detectors which can be simulated by perfect detectors together with beamsplitters. A detector click indicates a failure of the conditional quantum eraser while the absence of a click means that the quantum erasure (of predictability) has succeeded. The success rate will be limited by the degree of predictability. The quantum eraser visibility will further depend on the amount of concurrence in the initial system which limits the quality of the retrieved interference fringes.

The structure of the paper is as follows. In Section [I] we present the interferometric system and consider the general idea of conditional quantum erasing. We also discuss differences between the conditional quantum eraser and conventional quantum erasers. Inequalities are derived and compared for the conditional and conventional quantum eraser. We restrict our considerations to pure states of the entangled system in this section. In Section III we apply the general idea of conditional quantum erasing to the four-level atom interferometer and discuss various realizations of conditional quantum erasure in this system. We derive the success probability of erasure and discuss its relation to the concurrence in the initial (unsorted) system. We extend our results to cases when the initial system is not in a pure (entangled) state in Section IV and show the limitation on the performance of conditional quantum erasure due to the mixture. In particular, it will turn out that we can not reach the maximum visibility anymore. Finally, in Section V, we present some concluding remarks and discussions.

\section{CONDITIONAL QUANTUM ERASURE: GENERAL CONSIDERATION}

We first discuss and summarize the general idea of the conditional quantum eraser on the basis of a simple model in an attempt to prepare the reader for the arguments which follow. Suppose we are given two spatially separated two-photon sources, $A$ and $B$ ( see Fig. 2), that generate the following polarization-entangled photon state

$$
|\Psi\rangle_{A, B}=|\pi\rangle_{A} \otimes|\sigma\rangle_{A}+|\pi\rangle_{B} \otimes|\sigma\rangle_{B}
$$

where $\pi$ and $\sigma$ denotes different polarization and the indexes $A$ and $B$ denote the origin of the photons. We stress already here that the entanglement between the photons is an essential ingredient for the nonclassical features of the conditional quantum eraser. The coupled system (2.1) contains $\sigma$-polarized photons, forming the "interfering system", which are detected at an interference screen, and the $\pi$-polarized photons which establish the "environmental degrees of freedom" and whose detection may serve as, e.g. a which-path measurement. In the "environment" only those degrees of freedom are included which can be controlled by the experimentalist by, e.g., which-path measurements or quantum eraser sorting.

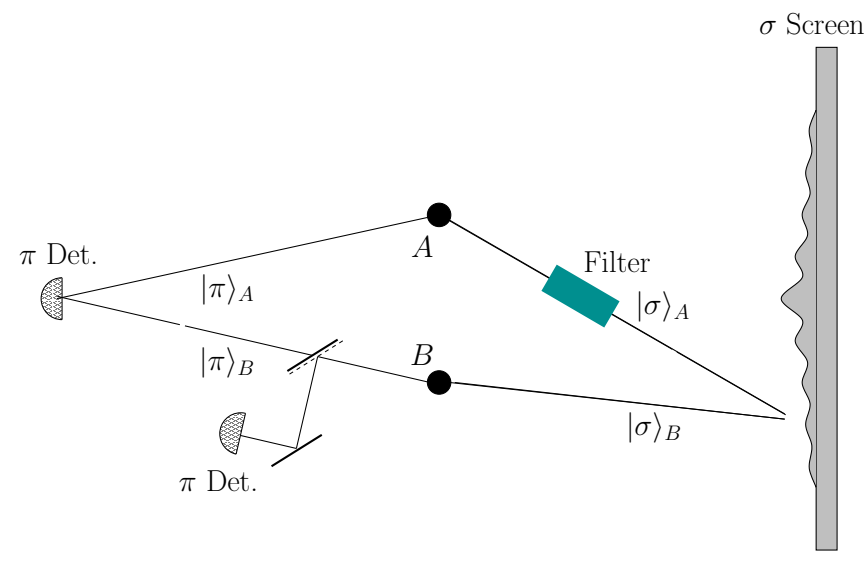

FIG. 2: Interferometric set-up of the conditional quantum eraser. An optical filter, with a transmittance $t$, in the upper right arm of the interferometer generates (partial) predictability about the path the interfering $\sigma$-photon takes. The measurement device in the lower left arm, consisting of a beamsplitter with transmittance $t_{\mathrm{BS}}$ and a photon detector, performs a partial, interaction-free which-path measurement on the erasing $\pi$-photon. If the detector clicks, the conditional quantum eraser failed. If the detector does not respond, the conditional quantum eraser succeeded. In this case the correlated measurement of the erasing photon at an equidistantly positioned detector in between the two atoms and the interfering photon will result in a complete erasing of the predictability under certain circumstances.

Let us first discuss the $\sigma$-polarized part of the system on the basis of the interference experiment displayed in Fig. 2. Suppose, we only consider the right part of the 
interference configuration in Fig. 2 which establishes a standard Young-type interference experiment with two atoms acting as the double-slit. First, we assume just one $\sigma$-polarized photon emitted by the two atoms and not the entangled photon system of Eq. (2.1). In addition, one of the two paths (the upper one in Fig. 2) is partially blocked by an optical filter. The intensity of the light or, to be more precise, the probability amplitude of the photon will be reduced, compared to the other optical path (the lower one in Fig. 2), as

$$
|\sigma\rangle=\frac{1}{\sqrt{1+t}}\left(\sqrt{t}|\sigma\rangle_{A}+|\sigma\rangle_{B}\right) .
$$

Here $t$ is the transmittance of the optical filter. The reduction obviously depends on the transmittance $t$. Consequently, partial, a priori knowledge about the path the photon actually takes is granted and this is expressed by the predictability $\mathcal{P}[13,14,15,16,17,18,19,20,21,22$, 23.

$$
\mathcal{P}=\left|\frac{1-t}{1+t}\right|=\frac{1-t}{1+t} .
$$

We expect the visibility $\mathcal{V}$ to be reduced as a consequence of this a priori knowledge in agreement with the general duality relation

$$
\mathcal{P}^{2}+\mathcal{V}^{2} \leq 1
$$

leading to the following maximum possible visibility in this interferometer

$$
\mathcal{V}_{\max }=\sqrt{1-\left(\frac{1-t}{1+t}\right)^{2}}=\frac{2}{1+t} \sqrt{t} .
$$

Let us now consider the complete interferometer including the environmental, $\pi$-polarized photon but neglecting the partial measurement device in the lower left path. The entanglement between the photons in Eq. 2.1) enables us to perform which-path measurement or quantum sorting on the environment, i.e. on the $\pi$-photon and this, in turn, alters the interference properties of the interference photon. In particular, as a consequence of the entanglement, we will not observe any interference fringes of the $\sigma$-photons if we do not detect the $\pi$-photon

$$
\begin{aligned}
\left\langle E_{A, \sigma}^{\dagger} E_{B, \sigma}+A \leftrightarrow B\right\rangle= & \left\langle\pi_{A} \mid \pi_{B}\right\rangle\left\langle\sigma_{A}\left|E_{A, \sigma}^{\dagger} E_{B, \sigma}\right| \sigma_{B}\right\rangle \\
& +A \leftrightarrow B \\
= & 0 .
\end{aligned}
$$

The entanglement between the subsystems enables us to get possible which-path information expressed by the quantity distinguishability, $\mathcal{D}$, which limits interference visibility according to the duality relation [17, 18]

$$
\mathcal{D}^{2}+\mathcal{V}^{2} \leq 1
$$

The distinguishability is the maximum possible whichpath information Nature can grant us and it is, in this interferometer, given by $\mathcal{D}=1$. This explains why we do not observe interference when we neglect the environment, i.e. do not detect or measure the $\pi$-photon. The actual knowledge, $\mathcal{K}$, which is the amount of which-path information that we can learn from the measurement of the environment, is usually smaller than the distinguishability, $\mathcal{D}$ 23. In particular, when we do not measure the $\pi$-photon, the actual which-way information $\mathcal{K}$ is just given by the a priori knowledge or predictability $\mathcal{P}$, Eq. (2.3), since we do not gain further information if we discount the environment. In general, the actual which-path information is limited by the following inequality [23]

$$
\mathcal{P} \leq \mathcal{K} \leq \mathcal{D} \text {. }
$$

One of the reasons, why we start with the entangled state, (2.1), is that quantum eraser sorting in the usual sense is immediately possible. This can be done, if we detect the environmental $\pi$-photon with a detector equidistantly placed from the two atoms as indicated in Fig. 2. A correlated detection of the $\sigma$ - and $\pi$-photons erases the distinguishability and we retrieve interference. However, the recovered quantum eraser visibility, $\mathcal{V}^{(\mathrm{QE})}$, of the interference fringes is limited if the a priori knowledge $\mathcal{P}$ is not equal to zero. The recovered quantum eraser visibility for the interferometer in Fig. 2 is given by

$$
\mathcal{V}^{(\mathrm{QE})}=\frac{2}{1+t} \sqrt{t}
$$

where $t$ is, again, the transmittance of the optical filter and where we took into account the following entangled state of the system under consideration

$$
|\Psi\rangle_{A, B}=\frac{1}{\sqrt{1+t}}\left(\sqrt{t}|\pi\rangle_{A} \otimes|\sigma\rangle_{A}+|\pi\rangle_{B} \otimes|\sigma\rangle_{B}\right) .
$$

In particular, the quantum eraser visibility is limited by the coherence which is, in the case of the pure entangled state under consideration, just the concurrence $\mathcal{C}=\frac{2}{1+t} \sqrt{t}$ [see Eq. (2.10] ] 28], and

$$
\mathcal{V} \leq \mathcal{V}^{(\mathrm{QE})} \leq \mathcal{C}
$$

Here $\mathcal{V}$ denotes the "a priori visibility" of the system before quantum erasing [23]. We note at this point, that the quantity $\mathcal{C}$ was also called the coherence in Ref. [23]. Although a possible relation of this quantity to an entanglement measure was mentioned, it was not associated with the concurrence introduced by Wootters [28]. Here, we close this gap by stating that the "coherence" is indeed a measure of entanglement which is explicitly given by the concurrence of the initial system. We also mention that the concurrence is directly connected with the two-particle visibility introduced by Jaeger et al. [18] (see also [29]) which gives the concurrence an evident physical meaning.

Englert and Bergou demonstrated the following erasure relation 23], on the basis of the inequalities (2.8) and (2.11),

$$
\mathcal{P}^{2}+\mathcal{C}^{2} \leq 1 .
$$


Thus, the maximum possible visibility of the recovered interference fringes, $\mathcal{V}_{\max }^{(\mathrm{QE})}$, can never reach unity if the predictability, $\mathcal{P}$, is not equal to zero,

$$
\mathcal{V}_{\max }^{(\mathrm{QE})} \leq \sqrt{1-\mathcal{P}^{2}}
$$

In other words, a conventional quantum eraser can never erase the a priori predictability $\mathcal{P}$. When we consult Eqs. (2.3) and (2.9) it is easy to see that the considered interferometric scheme satisfies the general inequalities, (2.12) and (2.13). Moreover, since the state under consideration is a pure state, the inequalities transform into equalities [23].

We have just showed that a conventional quantum eraser can not erase the a priori knowledge or predictability, $\mathcal{P}$. Does this mean that it is impossible to erase predictability? We anticipate the answer by claiming that it is indeed possible to erase predictability. However, this can only be achieved with a certain success probability which is subject to some constraints. We, therefore, call such an eraser a conditional quantum eraser. In the following, our task will be to find a device which erases the a priori knowledge. A naive approach starts, again, by considering the right part of the interferometric device in Fig. 2. We assume just a single $\sigma$-polarized photon which arises from the sources $A$ and $B$ and do not consider a biphoton at first. This approach will also help us to understand the crucial quantum features of the conditional quantum eraser. Suppose we utilize a second optical filter with the same transmittance, $t$, as the upper optical filter in the lower optical path of the right part of the interferometric device in Fig. 2. Accordingly, the initial state of the photon, (2.2), is transferred to

$$
|\sigma\rangle=\frac{1}{\sqrt{2 t}}\left(\sqrt{t}|\sigma\rangle_{A}+\sqrt{t}|\sigma\rangle_{B}\right) .
$$

Thus, we recover perfect visibility of the interference fringes and apparently erase the a priori knowledge. However, at the same time we change the a priori conditions of the interferometric setup. The second optical filter alters the interference device to an ideal two-way interferometer with no a priori knowledge about the path the photon takes. We have gained perfect fringe visibility by changing the interferometric device for the interfering $\sigma$-photon. In other words, we alter the a priori condition of the right part of the interferometer which defines the initial interferometric properties of the $\sigma$-photon. This, however, does not constitute the basis what we want to consider as an erasing of the a priori knowledge. In particular, we do not want to change the right part of the interferometric setup which alters the a priori conditions of the interfering $\sigma$-photon and consequently changes the a priori knowledge about the path the photon takes.

The concept to erase predictability without directly or locally affecting the interfering photon, in the sense of changing the a priori conditions of the right interferometer in Fig. 2, is realized by the quantum property entanglement. Thus, we have to consider a biphoton as, e.g. that of Eq. (2.1), for the arguments which follow. As already mentioned, entanglement between the photons is essential in order to meet the requirements for the conditional quantum eraser. Again, a naive approach starts with changing the a priori properties of the complete four-port interferometer in Fig. 2. That is, we insert an optical filter in the lower left arm of the interferometer with the same transmittance $t$ as the one in the upper right arm. Although classically this will not have any effect on the interfering $\sigma$-photon, quantum mechanically, due to the entanglement in the biphoton state (2.1), the biphoton is transformed into the following state

$$
|\Psi\rangle_{A, B} \rightarrow \frac{1}{\sqrt{2 t}}\left(\sqrt{t}|\pi\rangle_{A} \otimes|\sigma\rangle_{A}+\sqrt{t}|\pi\rangle_{B} \otimes|\sigma\rangle_{B}\right) .
$$

Consequently, we recover full interference fringes in a coincidence measurement of the two photons, i.e. under the quantum eraser conditions. However, the initial properties of the four-port interferometer have changed albeit under an intriguing, nonlocal and certainly nonclassical manner. The predictability of the path of the biphoton has become zero in Eq. (2.15) due to an optical intervention.

This is still not completely the situation which we want to consider as a conditional quantum eraser. We try to realize the conditional quantum eraser without changing the initial optical properties of the four-port interferometer. This can be achieved with the help of a nonlocal, partial and interaction-free which-way measurement of the path the photon takes 24]. As in the previous discussion, we assume a black box, representing the measurement device, instead of the optical filter in the lower left arm of the four-port interferometer. The measurement device will not locally interact with the interfering photon but only affects the "erasing photon". Thus, in the same manner as above, the entanglement between the interfering and the erasing photon plays an essential role in the manipulation of the optical properties of the interfering photon by the erasing photon. We will, at this point, not discuss details about the black box or measurement device but only argue about its general features. We assume a measurement device that can partly determine the path of the erasing photon ( $\pi$-photon) in the four-port interferometer. In addition, the measurement apparatus can report a positive or a negative result. That is, the measurement apparatus can unambiguously tell if it succeeded in partially distinguishing between the paths the $\pi$-photon took (positive result) or not (negative result). Further, the probability of failure is adjustable to the value predetermined by the predictability of the path of the interfering $\sigma$-photon, $1-t /(1+t)$. If we denote the states of the measurement device with $|0\rangle_{\mathrm{M}}$, related with a positive result or success, and $|1\rangle_{\mathrm{M}}$, connected with a negative result or failure, the state of the biphoton including the states of the measurement apparatus 
unitarily transforms into

$$
\begin{aligned}
|\Psi\rangle \rightarrow & \sqrt{\frac{1}{1+t}}\left[\left(\sqrt{t}|\sigma\rangle_{A} \otimes|\pi\rangle_{A}\right.\right. \\
& \left.+\sqrt{t}|\sigma\rangle_{B} \otimes|\pi\rangle_{B}\right) \otimes|0\rangle_{\mathrm{M}} \\
& \left.+\left(\sqrt{1-t}|\sigma\rangle_{B} \otimes|\pi\rangle_{B}\right) \otimes|1\rangle_{\mathrm{M}}\right] .
\end{aligned}
$$

This constitutes the initial state of the conditional quantum eraser. Suppose the measurement apparatus gives a positive result. Under this condition the state of the biphoton is transformed in such a way that we recover perfect fringe visibility for the $\sigma$-photon under quantum erasing conditions, i.e. in a coincidence measurement of the biphoton. The partial, interaction-free which-way measurement of the $\pi$-photon manipulates the entangled $\sigma$-photon in such a manner that full interference fringes are recovered in a quantum eraser scheme.

The partial interaction-free measurement becomes evident if we consider the effect of the measurement on the $\pi$-photon itself. Clearly, the initial state of the $\pi$-photon, $|\pi\rangle_{A}+|\pi\rangle_{B}$ is transformed into $|\pi\rangle_{A}+\sqrt{t}|\pi\rangle_{B}$ under the influence of the partial, interaction-free measurement. If, on the other hand, the measurement apparatus responds (negative result) we will discard the biphoton. In other words, we have erased the predictability or the a priori knowledge of the path the $\sigma$-photon takes with the help of an interaction-free, partial which-way measurement of the $\pi$-photon. However, this can only be achieved under the condition that the measurement apparatus does not react. The probability that this takes place is connected with the initial predictability of the $\sigma$-photon path. In particular the failure probability of our conditional quantum eraser is directly given by the predictability, $\mathcal{P}$. The realization of the measurement apparatus or the black box will be the subject of the next section. We will also make clear why we can talk about an interaction-free measurement.

\section{CONDITIONAL QUANTUM ERASURE IN A TWO-ATOM INTERFEROMETER}

We start with the consideration of the two-atom, fourport interferometer in Fig. 2. Suppose the initial atomic state is given as

$$
|\Psi\rangle_{\text {atom }}=|1\rangle_{A} \otimes|1\rangle_{B}
$$

Applying a $\pi$-polarized laser pulse with weak enough intensity that only one atom at any given time will be excited, the atoms transform into

$$
|\Psi\rangle_{\text {atom }} \rightarrow|1\rangle_{A} \otimes|3\rangle_{B}+|3\rangle_{A} \otimes|1\rangle_{B} .
$$

Let us assume that the detector on the right in our fourport interference scheme is only sensitive to $\sigma$-polarized photons, i.e. we ignore $\pi$-polarized photons. In this case the system evolves into the following state

$$
\begin{aligned}
|\Psi\rangle_{\text {atom }} & \rightarrow|\Psi\rangle_{\text {atom }} \otimes|\Psi\rangle_{\mathrm{ph}}, \\
& =|1\rangle_{A} \otimes|2\rangle_{B} \otimes|\sigma\rangle_{B}+|2\rangle_{A} \otimes|1\rangle_{B} \otimes|\sigma\rangle_{A},
\end{aligned}
$$

after decay of the atoms. Next, we apply a second, $\sigma^{+}$ circularly polarized laser pulse, again weak enough that only one atom at a time will be excited. This transforms the atomic state into $|\Psi\rangle_{\text {atom }}=|1\rangle_{A} \otimes|3\rangle_{B}+|3\rangle_{A} \otimes|1\rangle_{B}$. Now, we assume the detector in the left part of our fourport interferometer only to be sensitive to $\pi$-polarized photons. That is, we ignore $\sigma$-polarized photons in the second transition. Under this condition, after the decay of the atoms, the system will evolve into the following state

$$
\begin{aligned}
|\Psi\rangle_{\text {atom }} \otimes|\Psi\rangle_{\mathrm{ph}} \rightarrow & |1\rangle_{A} \otimes|1\rangle_{B} \otimes|\sigma\rangle_{B} \otimes|\pi\rangle_{B} \\
& +|1\rangle_{A} \otimes|1\rangle_{B} \otimes|\sigma\rangle_{A} \otimes|\pi\rangle_{A} .
\end{aligned}
$$

We ignored the time-dependence of the photon arrivals as well as the time-dependence of the application of the laser pulses throughout the above derivation for simplicity reasons. Further, the time-dependence of the photon arrivals is not important for the considerations which follow. We notice, that the two-atom interferometer can generate an entangled state between the photons identical to the entangled photon state in Eq. (2.1). From now on, we neglect the atomic subspace in (3.4) which will not change after generation of the biphoton state and reaches the initially assumed atomic state after each generation of biphotons, ready for another generation cycle.

Let us first neglect the measurement device in the lower left arm of the four-port interferometer in Fig. 2. The optical filter in the upper right arm of the interferometer generates a priori knowledge of the $\sigma$-polarized photon in dependence of its transmittance $t$. In particular, the $\sigma$-photon state by itself will be transformed into $|\sigma\rangle_{A}+|\sigma\rangle_{B} \rightarrow\left(\sqrt{t}|\sigma\rangle_{A}+|\sigma\rangle_{B}\right) / \sqrt{(1+t)}$ which clearly shows that the partial predictability of the path the photon takes is given by $\mathcal{P}=1-t /(1+t)$. Translating this to the biphoton state (3.4) and ignoring the atomic subspace we arrive at the expression,

$$
|\Psi\rangle_{\mathrm{ph}} \rightarrow \sqrt{\frac{1}{1+t}}\left(|\sigma\rangle_{B} \otimes|\pi\rangle_{B}+\sqrt{t}|\sigma\rangle_{A} \otimes|\pi\rangle_{A}\right) .
$$

In other words, in a coincidence measurement of the biphoton with the detector of the $\pi$-photon equidistantly placed from the two atoms we realize the conventional quantum eraser condition 5 . The ability to recover interference fringes is limited by the a priori knowledge about the path the $\sigma$-photon takes.

In order to implement the conditional quantum eraser we have to insert the measurement device. In particular, we want to realize a partial, interaction-free which-way 
measurement of the $\pi$-photon. A possible device capable in doing this is already implied in the lower left arm of the four-port interferometer, in Fig. 2. It consists of a beamsplitter which splits the path of the $|\pi\rangle_{B}$-photon into two alternatives. We place a detector, which operates with $100 \%$ efficiency, in one of the two alternatives. Suppose the ratio between transmission and reflection of the beamsplitter can be adjusted to a preset value. In particular, we can set the transmittance of the beamsplitter, $t_{\mathrm{BS}}$, equal to the transmittance of the optical filter, $t_{\mathrm{BS}}=t$, in the upper right arm of the interferometer.

Let us consider how this affects the $\pi$-photon, i.e. we ignore for a moment the $\sigma$-photon and consider the $\pi$ photon alone. Due to the unitary transformation of the beamsplitter, the $\pi$-photon transforms into the following state

$$
\begin{aligned}
\frac{1}{\sqrt{2}}\left(|\pi\rangle_{A}+|\pi\rangle_{B}\right) \rightarrow \frac{1}{\sqrt{2}}[ & \left(|\pi\rangle_{A}+\sqrt{t_{\mathrm{BS}}}|\pi\rangle_{B}^{(1)}\right) \otimes|0\rangle_{\mathrm{M}} \\
& \left.+\sqrt{1-t_{\mathrm{BS}}}|\pi\rangle_{B}^{(2)} \otimes|1\rangle_{\mathrm{M}}\right] .
\end{aligned}
$$

Here we took into account the detector states $|0\rangle_{\mathrm{M}}$, indicating success, and $|1\rangle_{\mathrm{M}}$ for failure (the detector clicks), as well as the two alternatives $|\pi\rangle_{B}^{(1)}$ and $|\pi\rangle_{B}^{(2)}$ of the $|\pi\rangle_{B}$-photon amplitude. The alternative $|\pi\rangle_{B}^{(1)}$, of course, is the original mode or the original "path" the $\pi$-photon takes. It is evident from Eq. (3.6) that the beamsplitter together with the detector performs a partial and interaction-free which-way measurement of the path the $\pi$-photon takes in the interferometer. In particular, when no interaction with the detector takes place, i.e. the detector is in the $|0\rangle_{\mathrm{M}}$ state which is the outcome corresponding to success, we gain partial information about the $\pi$-photon path. The obtained knowledge is given by $\mathcal{K}=\left(1-t_{\mathrm{BS}}\right) /\left(1+t_{\mathrm{BS}}\right)$. On the other hand, the partial measurement fails if the detector reacts, i.e. a photon is detected, indicated by the detector state $|1\rangle_{\mathrm{M}}$. In other words, a partial, interaction-free measurement of the $\pi$ photon takes place under the condition that the detector does not click. Consequently, we are allowed to speak about a conditional, interaction-free and partial whichway measurement.

Next, we consider the effect of the partial which-way measurement on the biphoton given by Eq. (3.5). The biphoton transfers into the following state as a consequence of the $\pi$-photon measurement

$$
\begin{aligned}
|\Psi\rangle_{\mathrm{ph}} \rightarrow & \sqrt{\frac{1}{1+t}}\left[\left(\sqrt{t_{\mathrm{BS}}}|\sigma\rangle_{B} \otimes|\pi\rangle_{B}+\sqrt{t}|\sigma\rangle_{A} \otimes|\pi\rangle_{A}\right)\right. \\
& \otimes|0\rangle_{\mathrm{M}} \\
& \left.+\sqrt{1-t_{\mathrm{BS}}}|\sigma\rangle_{B} \otimes|\pi\rangle_{B}^{(2)} \otimes|1\rangle_{\mathrm{M}}\right], \\
= & \sqrt{\frac{1}{1+t}}\left[\sqrt { t + t _ { \mathrm { BS } } } \left(\sqrt{\frac{t_{\mathrm{BS}}}{t+t_{\mathrm{BS}}}}|\sigma\rangle_{B} \otimes|\pi\rangle_{B}\right.\right. \\
& \left.+\sqrt{\frac{t}{t+t_{\mathrm{BS}}}}|\sigma\rangle_{A} \otimes|\pi\rangle_{A}\right) \otimes|0\rangle_{\mathrm{M}}
\end{aligned}
$$

$$
\left.+\sqrt{1-t_{\mathrm{BS}}}|\sigma\rangle_{B} \otimes|\pi\rangle_{B}^{(2)} \otimes|1\rangle_{\mathrm{M}}\right] .
$$

Here, we did not explicitly label alternative one of the $\pi$-photon since it is the original path, introduced previously. From Eq. (3.7) we see that it is possible to recover perfect fringe visibility of the $\sigma$-photon in a coincidence measurement with the $\pi$-photon detected at an equal distance from the two atoms. Thus, the "no-click" event in the measurement device indicates a "click" event on the eraser detector. This fact is important in practice if inefficient detectors are involved. In case of ideal conditions, we detect all involved photons and the "no-click" event on the measurement device is equivalent with a "click" event on the eraser detector. However, this can only be achieved when, first, the transmittance of the beamsplitter is equal to the transmittance of the optical filter, $t_{\mathrm{BS}}=t$, and, second, under the condition that the photon detector of the measurement apparatus does not respond, i.e. the measurement device works interaction free and resides in the state $|0\rangle_{\mathrm{M}}$. In other words we have implemented the conditional quantum eraser with the help of the partial, interaction-free measurement device. We have erased the predictability of the path the $\sigma$-photon takes. We further verify, that the failure probability of the partial measurement device is determined by the a priori predictability $\mathcal{P}=1-t /(1+t)$ of the path the $\sigma$-photon takes in case of optimal erasing conditions, i.e. when $t_{\mathrm{BS}}=t$. Moreover, the product of success probability and two-particle visibility or concurrence of the obtained quantum state, (3.7), is limited by the degree of concurrence or two-particle visibility of the initial state, (2.10) (see also discussion following Eq. (2.10)),

$$
\frac{t+t_{\mathrm{BS}}}{1+t} \frac{2 \sqrt{t t_{\mathrm{BS}}}}{t+t_{\mathrm{BS}}}=\frac{2 \sqrt{t t_{\mathrm{BS}}}}{1+t} \leq \mathcal{C}=\frac{2}{1+t} \sqrt{t},
$$

since $0 \leq t_{\mathrm{BS}} \leq 1$. In Eq. (3.8) the two-particle visibility or concurrence is given by $\mathcal{C}_{\text {cond }}=\frac{2 \sqrt{t t_{\mathrm{BS}}}}{t+t_{\mathrm{BS}}}$, and the probability of success is given by $S=\frac{t+t_{\mathrm{BS}}}{1+t}$. We mention, that the two-particle visibility of the obtained quantum state, (3.7), is identical to the "single-particle visibility" in the conditional quantum eraser, $\mathcal{V}_{\text {cond }}^{(\mathrm{QE})}=\mathcal{C}_{\text {cond }}$ of the $\sigma$-photon in case of quantum erasing condition, i.e. in a coincidence measurement of the biphoton with a $\pi$ photon detector equidistantly placed from the two atoms $A$ and $B$. We mention also, that the conditional quantum eraser visibility, $\mathcal{V}_{\text {cond }}^{(\mathrm{QE})}$, strongly depends on the transmittance $t_{\mathrm{BS}}$. When the conditional quantum eraser does not work optimally, i.e. $t_{\mathrm{BS}} \neq t$, the conditional quantum eraser visibility will be smaller than one,

$$
\mathcal{V}_{\text {cond }}^{(\mathrm{QE})}=\frac{2 \sqrt{t t_{\mathrm{BS}}}}{t+t_{\mathrm{BS}}}<1,
$$

and when the conditional quantum eraser works optimally, i.e. for $t_{\mathrm{BS}}=t$, the conditional quantum eraser visibility reaches one,

$$
\mathcal{V}_{\mathrm{cond}}^{(\mathrm{QE})}=\frac{2 \sqrt{t t_{\mathrm{BS}}}}{t+t_{\mathrm{BS}}}=1 .
$$


Equations (3.9) and 3.10), again, clearly indicate that the conditional quantum eraser visibility may exceed the quantum eraser visibility of a conventional quantum eraser. In particular, the conditional quantum eraser visibility can explicitly top the concurrence or two-particle visibility of the initial system which is impossible with a conventional quantum eraser [see Eq. (2.11)].

The complementarity relation 2.12) represents the main feature of the conditional quantum eraser. It contains all the important facets of the physical peculiarities. First, we get a direct relation between entanglement and complementarity. In particular, the concurrence is a well known entanglement measure [28] and it is, in the case of the four-port two-atom interferometer, given by the measurable quantity of two-particle visibility 18. A similar expression for real particles has also been found in [30, but only with the equal sign. In addition, the expression there contains distinguishability and not predictability, which arises because of the entanglement of the biparticle to an additional subsystem. In Eq. (2.12) the concurrence is related to a single particle property of one of the two biphotons, i.e. to the system itself in the form of predictability. Here, we will, however, not discuss the general properties and the general validity of this complementarity relation as an entanglement measure, but only some of its relevant characteristics with relation to the conditional quantum eraser. We will discuss a more general version of the complementarity relation with regard to entanglement measures and nonlocality or violations of Bell-type inequalities in another publication.

We want to verify that the general complementarity relation, Eq. (2.12), is fulfilled in the conditional quantum eraser. Although we can erase the a priori predictability of the $\sigma$-photon path, this does not mean that we can violate the general complementarity relation (2.12). The conditional quantum eraser can violate the quantum eraser relation for conventional quantum erasers, Eq. (2.13), but not the general complementarity relation. The conditional quantum eraser can change the initial conditions, $\mathcal{P}$ and $\mathcal{C}$, of the biphoton in a certain subspace of the complete biphoton plus detector Hilbert space determined by the null result of the detector. The ability to achieve this rearrangement in a conditional quantum eraser goes beyond the abilities of the conventional quantum eraser. The predictability, $\mathcal{P}_{\text {cond }}$, and concurrence, $\mathcal{C}_{\text {cond }}$, in the conditional quantum eraser, on condition that the detector is in the $|0\rangle_{\mathrm{M}}$ state, are given by

$$
\begin{aligned}
& \mathcal{P}_{\text {cond }}=\frac{\left|t-t_{\mathrm{BS}}\right|}{t+t_{\mathrm{BS}}} \\
& \mathcal{C}_{\text {cond }}=\frac{2 \sqrt{t_{\mathrm{BS}} t}}{t+t_{\mathrm{BS}}} .
\end{aligned}
$$

Clearly, the two complementary quantities, (3.11) and (3.12), fulfill the general complementarity relation, (2.13), for pure states

$$
\mathcal{P}_{\text {cond }}^{2}+\mathcal{C}_{\text {cond }}^{2}=\frac{\left(t-t_{\mathrm{BS}}\right)^{2}}{\left(t+t_{\mathrm{BS}}\right)^{2}}+\frac{4 t_{\mathrm{BS}} t}{\left(t+t_{\mathrm{BS}}\right)^{2}},
$$

$$
=1 \text {. }
$$

However, when $t=t_{\mathrm{BS}}$ we can fully erase the predictability and recover perfect fringe visibility clearly indicating the ability of the conditional quantum eraser to change the a priori conditions which limit the conventional quantum eraser. In the next section we consider effects of mixture on the conditional quantum eraser. We will expose additional limitations of the conditional quantum eraser which will be related to the concurrence of the initial system.

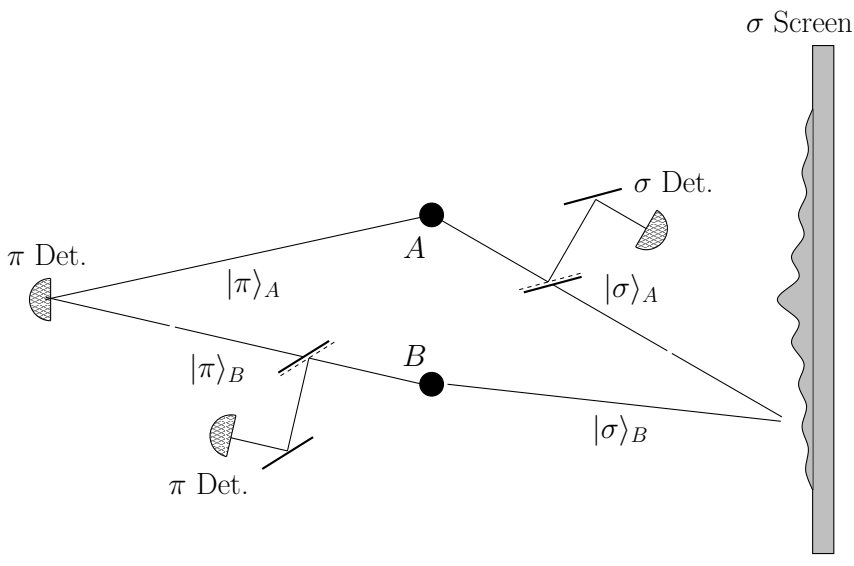

FIG. 3: Compared to the set-up in Fig. 2, this interferometer contains an additional partial interaction free which-way measurement in the upper right arm. It consists of a beamsplitter with transmittance $t_{1}$ and a $\sigma$-detector. Under the condition that the detector does not click, we gain which-path knowledge about the $\sigma$-photon path. This gained knowledge can be similarly erased as in Fig. 2. The additional partial which-path measurement device consists of a beamsplitter with transmittance $t_{2}$ and a $\pi$-photon detector.

First, we want to discuss a different conditional quantum eraser scheme, shown in Fig. 3. It combines two partial interaction-free measurements. The first one provides actual knowledge about the path the $\sigma$-photon takes, the second one erases the obtained which-path information. Here, we do not erase the a priori predictability about the path the $\sigma$-photon takes but the obtained knowledge from a conditional, interaction-free measurement. In a conventional quantum eraser, the actual which-path information sets a limit on the quantum eraser visibility. We can not erase the actual which-path knowledge, $\mathcal{K}$, obtained by the conditional, interaction-free measurement with the conventional quantum eraser and the maximum possible quantum eraser visibility, $\mathcal{V}_{\max }^{\mathrm{QE}}$, is given by

$$
\left(\mathcal{V}_{\max }^{\mathrm{QE}}\right)^{2} \leq 1-\mathcal{K}^{2} .
$$

In a conditional quantum eraser, however, we can erase the actual which-path knowledge with the help of a second, interaction-free measurement on the $\pi$-photons. Let us consider the effect of the first device which performs a partial which-way measurement. Suppose the beamsplitter has a transmittance of $t_{1}$. This affects the biphoton 
according to

$$
\begin{aligned}
|\Psi\rangle_{A, B}= & \frac{1}{\sqrt{2}}\left[\left(\sqrt{t_{1}}|\sigma\rangle_{A}|\pi\rangle_{A}+|\sigma\rangle_{B}|\pi\rangle_{B}\right) \otimes|0\rangle_{M 1}\right. \\
& \left.+\sqrt{1-t_{1}}\left(|\sigma\rangle_{A}^{(2)}|\pi\rangle_{A}\right) \otimes|1\rangle_{M 1}\right]
\end{aligned}
$$

where we take into account the detector states of the measurement device and denote the alternative way of the $\sigma_{A}$-photon with $|\sigma\rangle_{A}^{(2)}$. This photon, of course, will be absorbed when the detector clicks. When the detector does not click, an interaction-free, partial which-way measurement of the $\sigma$-photon is accomplished. Depending on the transmittance, $t_{1}$, we gain some knowledge about the path the $\sigma$-photon takes, given by

$$
\mathcal{K}=\frac{1-t_{1}}{1+t_{1}}
$$

The probability, that the detector does not click, i.e. the partial which-way measurement succeeds, is given by $\left(1+t_{1}\right) / 2$. The visibility in a conventional quantum eraser scheme, which consists of the correlated detection of the $\pi$ - and $\sigma$-photons with a $\pi$-detector equidistantly positioned in between the two atoms under the condition that the partial which-path measurement succeeds, is given by

$$
\mathcal{V}_{\max }^{\mathrm{QE}}=\frac{2 \sqrt{t_{1}}}{1+t_{1}} .
$$

Clearly, the conventional quantum eraser can not erase the which-path knowledge obtained by the partial whichway measurement. The retrieved quantum eraser visibility is limited by the obtained knowledge from the partial which-way measurement and the relation (3.14) is satisfied

$$
\left(\mathcal{V}_{\max }^{\mathrm{QE}}\right)^{2}=\frac{4 t_{1}}{\left(1+t_{1}\right)^{2}}=1-\mathcal{K}^{2}=1-\left(\frac{1-t_{1}}{1+t_{1}}\right)^{2} .
$$

On the other hand, the conditional quantum eraser which additionally performs an interaction-free, partial whichway measurement on the $\pi$-photons can erase the obtained knowledge. This, however, can only be achieved with a certain success probability which is limited by the obtained which-path knowledge from the first measurement. Assuming a transmittance of $t_{2}$ of the beamsplitter, the second, interaction-free partial which-way measurement on the $\pi$-photon transforms the state 3.15) into

$$
\begin{aligned}
|\Psi\rangle_{A, B}= & \frac{1}{\sqrt{2}}\left[\left(\sqrt{t_{1}}|\sigma\rangle_{A}|\pi\rangle_{A}+\sqrt{t_{2}}|\sigma\rangle_{B}|\pi\rangle_{B}\right)\right. \\
& \otimes|0\rangle_{M 1} \otimes|0\rangle_{M 2} \\
& +\left(\sqrt{1-t_{2}}|\sigma\rangle_{B}|\pi\rangle_{B}^{(2)}\right) \otimes|1\rangle_{M 2} \\
& \left.+\left(\sqrt{1-t_{1}}|\sigma\rangle_{A}^{(2)}|\pi\rangle_{A}\right) \otimes|1\rangle_{M 1}\right]
\end{aligned}
$$

The conditional quantum eraser can fully recover interference fringes with visibility one if the transmittances of the beamsplitters are identical, $t_{1}=t_{2}$. This, however, only happens with a probability of $\left(t_{1}+t_{2}\right) / 2$ in which case none of the detectors clicks. Clearly, the success probability goes to zero if the knowledge about the path the $\sigma$-photon takes reaches one, i.e. the transmittance $t_{1} \rightarrow 0$. Similarly to the previous scenario, the product of the success probability and the retrieved interference visibility is limited by the concurrence of the biphoton system after the first interaction-free which-way measurement, which is given by

$$
\mathcal{C}=\sqrt{t_{1}}
$$

The conditional quantum eraser can erase the partial which-way information and fully recover interference fringes as long as the biphoton system after the first which-path measurement contains some concurrence, i.e. $\sqrt{t_{1}}>0$. It is the (additional) non-unitary evolution initiated by the partial which-way measurement which enables us to recover the full interference fringes in a conditional quantum eraser. This (additional) non-unitary evolution is the reason why the conditional quantum eraser outperforms the conventional or traditional quantum eraser. We note that the correlated measurement of the $\pi$ - and $\sigma$-photons represents also a nonunitary evolution which both the conventional and conditional quantum eraser have in common. It is this additional nonunitary evolution, induced by the partial which-way measurement in the conditional quantum eraser, that leads to performance enhancement.

We stress that the interaction free measurement device consists of absolutely ideal elements. This is certainly not the case in praxis and limits the practical value of our model. In case of non-ideal detectors and beamsplitters there can be photon absorption in the beamsplitter and, consequently, the detectors will not fire. In addition, the detector efficiency in real experiment is relatively low and the overall efficiency of the conditional quantum eraser is strongly reduced. On the other side, the conditional quantum eraser is based on the detection of all involved photons in an intensity correlation experiment. Therefore, whenever photons are lost in case of absorption at the beamsplitters or inefficient detectors, these events will not contribute to the intensity correlation function. The no-click event in the measurement device is, ideally, a click event in the eraser detector. In other words, all involved photon are ideally detected, since detectors are placed in both alternatives of the beamsplitters. Consequently, inefficient detectors simply reduce the overall efficiency of the conditional quantum eraser. Absorption in the beamsplitters, however, changes the ideal partial which way information to an effective partial which way information which depends on the degree of absorption. This must be taken into account in realistic experiments and appropriate modifications are necessary. These modifications are absorption devices in the other optical paths in order to balancing the losses. 


\section{EFFECTS OF MIXTURE IN THE INITIAL SYSTEM}

In this section we address the problem if and how some mixture in the initial two-photon state affects the performance of the conditional quantum eraser. We study further its effects on the complementarity relation between entanglement or concurrence and predictability. In order to do this we consider the concurrence of a specifically prepared two-photon system and discuss its connection to the measurable two-particle visibility. Suppose we are given the following initial two-photon density operator

$$
\begin{aligned}
\rho_{\text {init }}= & \frac{1}{1+t}\left[t\left|\pi_{A}\right\rangle\left\langle\pi_{A}|\otimes| \sigma_{A}\right\rangle\left\langle\sigma_{A}\right|\right. \\
& +\left|\pi_{B}\right\rangle\left\langle\pi_{B}|\otimes| \sigma_{B}\right\rangle\left\langle\sigma_{B}\right| \\
& \left.+M \sqrt{t}\left(\left|\pi_{A}\right\rangle\left\langle\pi_{B}|\otimes| \sigma_{A}\right\rangle\left\langle\sigma_{B}\right|+A \leftrightarrow B\right)\right],
\end{aligned}
$$

where $M$ is a "coherence factor" bounded between $0 \leq$ $C \leq 1$ and can be considered as a measure of mixture in the initial two-photon state. The two-photon state (4.1) resembles the two-photon state (3.5) of the previous section except for the reduced coherence expressed by the coherence factor $M$. Thus, the biphoton state (4.1) can be produced by the two-atom interferometer in Fig. 2 and the reduced coherence can be generated with the help of the entangled atom system. In particular, we assume a decoherence process on the atoms which become entangled to the $\sigma$-photon during the generation of the photon system [see Eq. (3.3)]. Such a decoherence process can be induced by, e.g. a measurement process which determines the population in the atomic ground-states, $|1\rangle$ and $|2\rangle$, of one of the two atoms [5]. Therefore, we may treat the coherence factor $M$ as the effect of a decoherence process on the atomic system during the generation of the biphoton in the interferometer, shown in Fig. 2. The two extremes, $M=1$ and $M=0$, correspond to a pure state and a fully incoherent mixed state, respectively.

Let us first investigate the predictability, the twoparticle visibility and its relation to the concurrence of the given biphoton state, (4.1). These quantities play a crucial role in the conventional as well as in the conditional quantum eraser as already emphasized in the previous section. We are further interested to what extent the degree of mixture influences the complementarity relation between concurrence and predictability. Interestingly, the degree of coherence, $M$, does not have any effect on the predictability, $\mathcal{P}$. The predictability, $\mathcal{P}$, is completely determined by the presetting of the interferometer, i.e. the degree of transmittance $t$ of the optical filter in Fig. 2 (or the transmittance $t_{1}$ of the beamsplitter in Fig. 3 when we employ a partial, interactionfree which-path measurement in order to gain knowledge about the photon path),

$$
\mathcal{P}=\frac{1}{1+t}|1-t|=\frac{1-t}{1+t} .
$$

We turn our attention to the two-particle visibility, $\mathcal{V}_{12}(\pi, \sigma)$, in the initial biphoton system (4.1) which is defined as 18]

$$
\mathcal{V}_{12}(\sigma, \pi)=\frac{\left[\bar{G}_{\sigma \pi}^{(2)}(\vec{r}, \vec{\rho})\right] \max -\left[\bar{G}_{\sigma \pi}^{(2)}(\vec{r}, \vec{\rho})\right]_{\min }}{\left[\bar{G}_{\sigma \pi}^{(2)}(\vec{r}, \vec{\rho})\right] \max +\left[\bar{G}_{\sigma \pi}^{(2)}(\vec{r}, \vec{\rho})\right]_{\min }} .
$$

Here

$$
\begin{aligned}
\bar{G}_{\sigma \pi}^{(2)}(\vec{r}, \vec{\rho})= & G_{\sigma \pi}^{(2)}(\vec{r}, \vec{\rho})-G_{\sigma}^{(1)}(\vec{r}) G_{\pi}^{(1)}(\vec{\rho}) \\
& +\left|\vec{\Psi}_{\sigma}(\vec{r})\right|^{2}\left|\vec{\Psi}_{\pi}(\vec{\rho})\right|^{2},
\end{aligned}
$$

and $G_{\sigma \pi}^{(2)}=\left\langle I_{\sigma}(\vec{r}) I_{\pi}(\vec{\rho})\right\rangle$ and $G_{\sigma, \pi}^{(1)}=\left\langle I_{\sigma}(\vec{r})\right\rangle,\left\langle I_{\pi}(\vec{\rho})\right\rangle$ are second- and first-order intensity correlation functions. The factors $\left|\vec{\Psi}_{\sigma}(\vec{r})\right|^{2}$ and $\left|\vec{\Psi}_{\pi}(\vec{\rho})\right|^{2}$ express the intensity factors of the dipole radiation,

$$
\vec{E}_{A, B}^{(+) i}(\vec{r}, t)=\Theta\left(t-\frac{\left|\vec{r}_{A, B}\right|}{c}\right) \vec{\Psi}_{i}(\vec{r}) \sigma_{A, B}^{(-) i}\left(t-\frac{\left|\vec{r}_{A, B}\right|}{c}\right),
$$

where

$$
\vec{\Psi}_{i}(\vec{r})=\frac{-\mu \omega_{o}^{2}}{4 \pi r^{3} \epsilon_{o} c^{2}}\left[\left(\overrightarrow{\hat{\epsilon}_{i}} \times \vec{r}\right) \times \vec{r}\right] .
$$

Here, we have introduced the retarded times $t-\left|\vec{r}_{A, B}\right| / c$ for the radiation from atoms $A$ and $B$, respectively. Further, $\overrightarrow{\hat{\epsilon}}_{i}$ forms one of the unit vectors $\overrightarrow{\hat{x}}$, $\overrightarrow{\hat{y}}$ or $\overrightarrow{\hat{z}}$, depending on the direction of the dipole moment in question, and $\sigma_{A, B}^{(-) i}\left(t-\left|\vec{r}_{A, B}\right| / c\right)$ are the ordinary atomic lowering operators for atom $A$ and $B$ corresponding to the polarization direction $i$. Equation (4.4) contains a correction to the intensity correlation $\bar{G}_{\sigma \pi}^{(2)}$ resulting from the fact that non-correlated or disentangled photon pairs may also contribute to the second-order correlation function and this contribution must be subtracted. The additional correction, which is proportional to the product of constant overall intensities $\left|\vec{\Psi}_{\sigma}(\vec{r})\right|^{2}\left|\vec{\Psi}_{\pi}(\vec{\rho})\right|^{2}$, must be added back in, to compensate for excessive subtraction. The excessive subtraction is inherent in the expression $G_{\sigma \pi}^{(2)}-G_{\sigma}^{(1)} G_{\pi}^{(1)}$ for entangled biphotons [18]. The additional correction makes the function (4.3) positive definite or, to be more precise, the two-particle visibility lies within the interval $[0,1]$ as it should for a proper definition of visibility.

Taking into account the above definitions, we obtain the following result for the two-particle visibility of the initial biphoton state (4.1),

$$
\mathcal{V}_{12}(\sigma, \pi)=2 M \frac{\sqrt{t}}{1+t} .
$$

The effect of the coherence factor $M$ is obviously. In the case of a completely mixed initial state, $M=0$, we can no longer observe any two-particle visibility even when there is no a priori predictability, $\mathcal{P}=0$, which corresponds to 
$t=1$. Let us investigate the relation of two-particle visibility to the concurrence, $\mathcal{C}$, in the initial system. In principle it is clear, that two-particle visibility must have a relation to entanglement. Two-particle visibility is an explicit manifestation of the phase relation between the photons in a biphoton system. The concurrence of a bipartite, mixed density operator, $\rho$, is defined as 28]

$$
\mathcal{C}(\rho)=\max \left\{0, \lambda_{1}-\lambda_{2}-\lambda_{3}-\lambda_{4}\right\},
$$

where the $\lambda_{i}$ 's are the square roots of the eigenvalues of $\rho \tilde{\rho}$ in descending order. Here $\tilde{\rho}$ results from applying the spin-flip operation to $\rho^{*}$,

$$
\tilde{\rho}=\left(\sigma_{y} \otimes \sigma_{y}\right) \rho^{*}\left(\sigma_{y} \otimes \sigma_{y}\right),
$$

where $\sigma_{y}$ is the Pauli operator in the relevant standard basis and $\rho^{*}$ is the complex conjugation of $\rho$. The relevant basis states in the case of the biphoton, (4.1), are $|\pi\rangle_{A} \equiv|0\rangle$ and $|\pi\rangle_{B} \equiv|1\rangle$ for the $\pi$-photon system, and $|\sigma\rangle_{A} \equiv|0\rangle$ and $|\sigma\rangle_{B} \equiv|1\rangle$ for the $\sigma$-photon system. With this definition we arrive at the following result for the concurrence of the initial biphoton state (4.1),

$$
\mathcal{C}\left(\rho_{\text {init }}\right)=2 M \frac{\sqrt{t}}{1+t},
$$

which is identical to the measurable two-particle visibility (4.7). We note that a similar relation between concurrence and two-particle visibility was found in 29.

Let us finally discuss the impact of the mixture on the complementarity relation between predictability and concurrence or, equivalently, between predictability and two-particle visibility before we consider the conditional quantum eraser. We already indicated in the previous section that the equal sign in the complementarity relation can hold only if the biphoton is in a pure state. In the case of a mixed state, (4.1), the following complementarity relation between concurrence and predictability is found

$$
\begin{aligned}
\mathcal{C}^{2}\left(\rho_{\text {init }}\right)+\mathcal{P}^{2}\left(\rho_{\text {init }}\right) & =\frac{(1-t)^{2}}{(1+t)^{2}}+4 M^{2} \frac{t}{(1+t)^{2}}, \\
& =\frac{(1-t)^{2}}{(1+t)^{2}}+M^{2}\left[1-\frac{(1-t)^{2}}{(1+t)^{2}}\right], \\
& =\mathcal{P}^{2}\left(\rho_{\text {init }}\right)+M^{2}\left[1-\mathcal{P}^{2}\left(\rho_{\text {init }}\right)\right] .
\end{aligned}
$$

Thus, the concurrence or equivalently the two-particle visibility is bounded by the coherence factor $M$

$$
\mathcal{C}\left(\rho_{\text {init }}\right) \leq M
$$

The concurrence can not reach the optimal value of one even when the predictability in the initial biphoton system is equal to zero (when $t=1$ ) if the degree of coherence or, equivalently, the degree of purity is smaller than one $M<1$. We will see that this upper bound on the two-particle visibility or concurrence limits the performance of the conditional quantum eraser. The com-

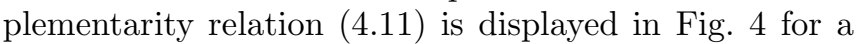
decoherence factor of $M=0.5$. The influence of the decoherence factor $M$ on the upper bound of the complementarity relation as well as on the concurrence is clearly recognizable.

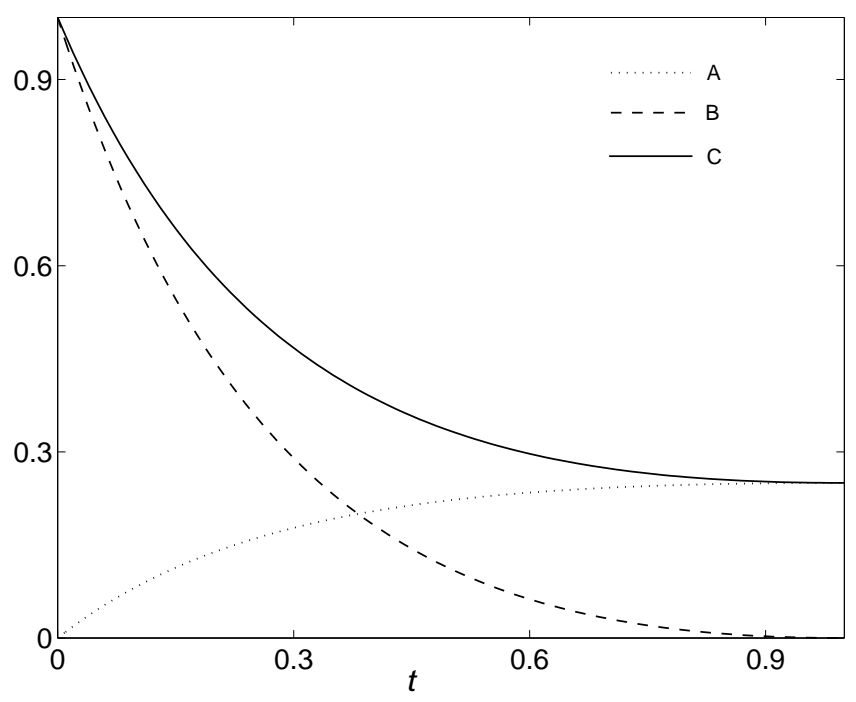

FIG. 4: Complementarity relation between concurrence or two-particle visibility and predictability for a mixed initial biphoton state vs. the transmittance $t$ of the optical filter. The coherence factor $M$ is given by $M=1 / 2$ and $A=\mathcal{C}^{2}\left(\rho_{\text {init }}\right), B=\mathcal{P}^{2}\left(\rho_{\text {init }}\right)$ and $C=\mathcal{C}^{2}\left(\rho_{\text {init }}\right)+\mathcal{P}^{2}\left(\rho_{\text {init }}\right)$.

We implement the measurement device in the lower left arm of the interferometer in Fig. 2, in order to realize the conditional quantum eraser. As in the previous section, the biphoton state, which becomes coupled to the state of the measurement device, transforms into

$$
\begin{aligned}
\rho_{\mathrm{ph}} & \otimes \rho_{\mathrm{M}}=\frac{t+t_{\mathrm{BS}}}{1+t}\left[\frac{t}{t+t_{\mathrm{BS}}}\left|\pi_{A}\right\rangle\left\langle\pi_{A}|\otimes| \sigma_{A}\right\rangle\left\langle\sigma_{A}\right|\right. \\
& +\frac{t_{\mathrm{BS}}}{t+t_{\mathrm{BS}}}\left|\pi_{B}\right\rangle\left\langle\pi_{B}|\otimes| \sigma_{B}\right\rangle\left\langle\sigma_{B}\right| \\
& \left.+\frac{M \sqrt{t_{\mathrm{BS}} t}}{t+t_{\mathrm{BS}}}\left(\left|\pi_{A}\right\rangle\left\langle\pi_{B}|\otimes| \sigma_{A}\right\rangle\left\langle\sigma_{B}\right|+A \leftrightarrow B\right)\right] \\
& \otimes\left|0_{\mathrm{M}}\right\rangle\left\langle 0_{\mathrm{M}}\right| \\
& \frac{1-t_{\mathrm{BS}}}{1+t}\left(\left|\pi_{B}^{(2)}\right\rangle\left\langle\pi_{B}^{(2)}|\otimes| \sigma_{B}\right\rangle\left\langle\sigma_{B}\right|\right) \otimes\left|1_{\mathrm{M}}\right\rangle\left\langle 1_{\mathrm{M}}\right|
\end{aligned}
$$

Here, again, $t_{\mathrm{BS}}$ is the transmittance of the beamsplitter of the measurement device and $\pi_{B}^{(2)}$ signifies the alternative path of the $\left|\pi_{B}\right\rangle$-photon due to the beamsplitter. The conditional quantum erasing succeeds if the photon detector of the measurement device does not click. In this case we realize an interaction-free partial which-way measurement of the $\pi$-photon path. It is also clear that the $\pi_{B}^{(2)}$-photon is absorbed if the measurement fails, i.e. the 
detector clicks. In contrast to the conditional quantum eraser of the previous section, which started with a pure entangled biphoton, the visibility of the retrieved interference fringes under quantum erasing conditions is now bounded by the coherence factor $M$. We stress again, that we understand the quantum eraser condition as a correlated measurement of the biphoton with an equidistantly placed photon detector in between the two atoms for the $\pi$-photon. Thus, the visibility we are talking about in the quantum eraser is a single-particle property of the $\sigma$-photon. The mixture in the initial state does not have an impact on the success or failure probability of the conditional quantum erasing, see Eqs. 3.7) and (3.8). However, the maximum possible conditional quantum eraser visibility (realized if $t_{\mathrm{BS}}=t$ ) is bounded by the coherence factor

$$
\mathcal{V}_{\text {cond }}^{(\mathrm{QE})}=M \frac{2 \sqrt{t t_{\mathrm{BS}}}}{t+t_{\mathrm{BS}}} \leq \mathcal{V}_{\text {cond }}^{(\mathrm{QE}, \max )}=M
$$

Comparing this result with Eq. 4.12 we conclude that the concurrence and hence the two-particle visibility limits the retrieved conditional quantum eraser visibility. However, we emphasize that the retrieved conditional quantum eraser visibility exceeds the quantum eraser visibility of the conventional quantum eraser $\mathcal{V}_{\max }^{(\mathrm{QE})}$ which is limited by

$$
\mathcal{V}_{\max }^{(\mathrm{QE})} \leq M \sqrt{1-\mathcal{P}^{2}}
$$

Thus, whenever the a priori knowledge about the path the $\sigma$-photon takes is unequal zero, the visibility of the retrieved interference fringes in the conditional quantum eraser tops that of the conventional quantum eraser. In other words the complementarity relation between concurrence and predictability which forms a physical statement of two-particle properties crucially affects singleparticle features of one of the two particles.

Interestingly, the product of the success probability and the visibility of the recovered interference fringes in the conditional quantum eraser is bounded by Eq. 4.15) as the visibility by itself is in the conventional quantum eraser. This clearly demonstrates how the conditional quantum eraser operates. The conditional quantum eraser works successfully if and only if the detector of the partial which-way measurement device does not click. In other words, a non-unitary projection onto a certain subspace takes place. We mention finally, that the above consideration can easily be transferred to the interference device in Fig. 3 similar as in the previous section. The only difference is the which-path knowledge gained from the additional measurement device for the $\sigma$-photon instead of the a priori which-path knowledge.

\section{CONCLUSIONS}

The usual quantum eraser problem considers the erasure of possible but not actual which-way information.
Here, we have considered a different task. We study a conditional quantum eraser to erase a priori or actual which-path information. The conditional quantum eraser employs a partial, interaction-free measurement on the erasing photon which crucially influences on the interfering photon if the two photons form an entangled state. The conditional quantum eraser erases the a priori knowledge of the interfering photon completely. However, this can only be achieved with a certain success probability which explains the conditional operation. Thus, the conditional quantum eraser constitutes a nonunitary transformation on the biphoton. The visibility of the retrieved interference fringes exceeds that of a conventional quantum eraser which is bounded according to a complementarity relation between concurrence or twoparticle visibility and predictability. In particular, the visibility of the recovered interference fringes in a conventional quantum eraser can not exceed a certain limit set by the a priori knowledge about the path the interference photon takes. In other words, a conventional quantum eraser can not erase predictability or actual which-path information. On the other hand, the complementarity relation between the concurrence and predictability limits the performance of the conditional quantum eraser, as well. Especially in cases when the initial biphoton system contains some degree of mixture, the visibility of the retrieved interference fringes can not exceed the upper bound of the inequality set by the complementarity relation. This generates an interesting scenario where two-particle properties crucially influence singleparticle properties. The complementarity relation between concurrence and predictability opens an interesting area of investigations which focuses on connections between complementarity and nonlocality. In this context we note that a similar complementarity relation was found in 30]. However, the relation between concurrence and the observable two-particle visibility as well as the influences of mixture in the biparticle was not investigated there.

In conclusion, we have studied the conditional quantum eraser in a two-atom, four-port interferometer. The two atoms substitute a Young-type double-slit an generate an entangled photon pair. The two photons are orthogonally polarized and thus distinguishable. One of the photons is detected at an interference screen (the interference photon) while the other one is detected at an equidistant position from the two atoms (the erasing photon). This establishes the conventional quantum eraser scenario. The conditional quantum eraser additionally performs a partial and interaction-free which-path measurement on the erasing photon. The interaction-free, partial which-path measurement can be simulated with a beamsplitter and an additional detector. The detector indicates whether the conditional quantum eraser has succeeded or failed. In particular, a detector click results in a failure of the conditional quantum eraser. The entangled atom-photon system, during the generation of the biphoton, enables us to study effects of mixedness in the bipho- 
ton state on the performance of the conditional quantum eraser. In addition, effects on the complementarity relation between two-particle visibility and predictability can be explored. A measurement process on the atoms which introduces decoherence effects [5] may be employed in order to generate a certain amount of mixture in the biphoton system. Finally, we note that the Young-type interference experiment was realized by Eichmann et al. 26] and the implementation of a conditional quantum eraser should be experimentally feasible.

\section{Acknowledgments}

We acknowledge helpful discussions with Y. Abranyos, M. Hillery, I. Nemeth, S. Stenholm, and Y. Sun. This research was supported by a grant from the Office of Naval Research (grant No. N00014-92J-1233), by the European Union Research and Training Network COCOMO, Contract No. HPRN-CT-1999-00129 and by a grant from PSC-CUNY.
[1] M. O. Scully and K. Drühl, Phys. Rev. A 25, 2208 (1982).

[2] N. Bohr, Naturwissenschaften 16, 245 (1928); Nature (London) 121, 580 (1928).

[3] M. Hillery and M. O. Scully, Quantum Optics, Experimental Gravity, and Measurement Theory (Plenum, NY, 1983) p. 65.

[4] M. O. Scully, B. G. Englert, and H. Walther, Nature (London) 351, 111 (1991).

[5] Y. Abranyos, M. Jakob, and J. Bergou, Phys. Rev. A 60 R2618 (1999); Acta Phys. Slov. 48, 255 (1998).

[6] M. Jakob, Y. Abranyos, and J. A. Bergou, Phys. Rev. A 64, 062102 (2001).

[7] A. G. Zajonc, L. J. Wang, X. Y. Zou, and L. Mandel, Nature (London) 353, 507 (1991).

[8] P. G. Kwiat, A. M. Steinberg, and R. Y. Chiao, Phys. Rev. A 11, 7729 (1992).

[9] Th. J. Herzog, P. G. Kwiat, H. Weinfurter, and A. Zeilinger, Phys. Rev. Lett. 75, 3034 (1995).

[10] T. B. Pittman, D. V. Strekalov, A. Migdall, M. H. Rubin, A. V. Sergienko, and Y. H. Shih, Phys. Rev. Lett. 77, 1917 (1996).

[11] S. Dürr, T. Nonn, and G. Rempe, Nature (London) 395 , 33 (1998).

[12] Y.-H. Kim R. Yu, S. P. Kulik, Y. Shih, and M. O. Scully, Phys. Rev. Lett. 84, 1 (2000).

[13] W. K. Wooters and W. H. Zurek, Phys. Rev. D 19, 473 (1979).

[14] D. M. Greenberger and A. Yasin, Phys. Lett. A 128, 391 (1988).

[15] L. Mandel, Opt. Lett. 161882 (1991).

[16] H. Rauch and J. Summhammer, Phys. Lett. A 104, 44 (1984); J. Summhammer, H. Rauch, and D. Tuppinger, Phys. Rev. A 36, 4447 (1987).

[17] B. G. Englert, Phys. Rev. Lett. 77, 2154 (1996).

[18] G. Jaeger, A. Shimony, and L. Vaidman, Phys. Rev. A 51, 54 (1995).

[19] S. Dürr, T. Nonn, G. Rempe, Phys. Rev. Lett. 81, 5705 (1998).
[20] T. Tsegaye, G. Björk, A. Atatüre, A. V. Sergienko, B. E. A. Saleh, And M. C. Teich, Phys. Rev. A 62, 032106 (2000).

[21] G. Björk and A. Karlsson, Phys. Rev. A 58, 3477 (1998).

[22] Y. Abranyos, M. Jakob, and J. Bergou, Phys. Rev. A 61, 013804 (2000).

[23] B.-G. Englert and J. A. Bergou, Opt. Commun. 179, 337 (2000).

[24] A. C. Elitzur and S. Dolev, Phys. Rev. A 63, 062109 (2001).

[25] D. Polder and M. F. H. Schuurmans, Phys. Rev. A 14, 1468 (1976).

[26] U. Eichmann, J. C. Bergquist, J. J. Bollinger, J. M. Gilligan, W. M. Itano, D. J. Wineland, and M. G. Raizen, Phys. Rev. Lett. 70, 2359 (1993); W. M. Itano, J. C. Bergquist, J. J. Bollinger, D. J. Wineland, U. Eichmann, and M. G. Raizen, Phys. Rev. A 57, 4176 (1998).

[27] see for example: P. Kochan, H. J. Carmichael, P. R. Morrow, and M. G. Raizen, Phys. Rev. Lett. 75, 45 (1995); R. G. Brewer, ibid. 77, 5153 (1996); T. Wong, S. M. Tan, M. J. Collett, and D. F. Walls, Phys. Rev. A 55, 1288 (1997); G. M. Meyer and G. Yeoman, Phys. Rev. Lett. 79, 2650 (1997); P. D. D. Schwindt, P. G. Kwiat, and B.G. Englert, Phys. Rev. A 60, 4285 (1999); H. T. Dung and K. Ujihara, Phys. Rev. Lett. 84, 254 (2000); A. Luis, Phys. Rev. A 64, 012103 (2001); C. Schön and A. Beige, ibid. 64, 023806 (2001); C. Skornia, J. von Zanthier, G. S. Agarwal, E. Werner, and H. Walther, ibid. 64, 063801 (2001); G. S. Agarwal, J. von Zanthier, C. Skornia, and H. Walther, ibid. 65, 053826 (2002).

[28] see for example: W. K. Wootters, Quantum Inf. Comp. 1, 27 (2001).

[29] A. F. Abouraddy, B. E. A. Saleh, A. V. Sergienko, and M. C. Teich, Phys. Rev. A 64, 050101(R) (2001).

[30] S. Bose and D. Home, Phys. Rev. Lett. 88, 050401, (2002). 